\title{
Faddeev-Popov matrix in linear covariant gauge: First results
}

\author{
Attilio Cucchieri, ${ }^{1, *}$ David Dudal, ${ }^{2,3, \dagger}$ Tereza Mendes, ${ }^{1, \hbar}$ Orlando Oliveira, ${ }^{4, \S}$ Martin Roelfs, ${ }^{2, \|}$ and Paulo J. Silva ${ }^{4, \boldsymbol{\Phi}}$ \\ ${ }^{1}$ Instituto de Física de São Carlos, Universidade de São Paulo, \\ C.P. 369, 13560-970 São Carlos, São Paulo, Brazil \\ ${ }^{2}$ KU Leuven Kulak, Department of Physics, Etienne Sabbelaan 53 bus 7657, 8500 Kortrijk, Belgium \\ ${ }^{3}$ Ghent University, Department of Physics and Astronomy, Krijgslaan 281-S9, 9000 Gent, Belgium \\ ${ }^{4}$ CFisUC, Departamento de Física, Universidade de Coimbra, 3004-516 Coimbra, Portugal
}

(Received 25 September 2018; published 28 November 2018)

\begin{abstract}
We discuss a possible definition of the Faddeev-Popov matrix for the minimal linear covariant gauge on the lattice and present first results for the ghost propagator. We consider Yang-Mills theory in four spacetime dimensions, for $\mathrm{SU}(2)$ and $\mathrm{SU}(3)$ gauge groups.
\end{abstract}

DOI: 10.1103/PhysRevD.98.091504

\section{INTRODUCTION}

The behavior of Green's functions in the infrared limit of Yang-Mills theory has been studied extensively in Landau gauge, both analytically and numerically [1-3]. However, since the evaluation of propagators and vertices depends on the gauge condition, a natural extension of these works would be to consider the linear covariant gauge (LCG), which depends on a gauge-fixing parameter $\xi$ and has the Landau gauge as a limiting case, corresponding to $\xi=0$. On the lattice, there have been a few studies $[4,5]$ of the gluon propagator $D(p)$ in the LCG. These numerical data seem to agree with several analytic predictions [6-8], e.g., the transverse component of $D(p)$ is similar to the Landau case, with $D(0)$ decreasing when the gauge-fixing parameter $\xi$ increases. On the other hand, for the ghost propagator $G(p)$, there is a wide range of different analytic predictions. Indeed, the ghost dressing function $p^{2} G(p)$ has been predicted to be flat (and nonzero) in the infrared limit [9] or to be suppressed at small momenta when $\xi$ increases [6] or to be null at $p=0[7,10]$. Numerical results for $G(p)$, however, are not yet available, since a lattice definition of the Faddeev-Popov (FP) matrix, corresponding to the minimal LCG on the lattice [5], has not been implemented so far.

In this work, we define the FP matrix in the lattice minimal LCG by considering the quadratic expansion of

\footnotetext{
*attilio@ifsc.usp.br

tavid.dudal@kuleuven.be

${ }^{\ddagger}$ mendes@ifsc.usp.br

§orlando@fis.uc.pt

"martin.roelfs@kuleuven.be

qpsilva@teor.fis.uc.pt
}

Published by the American Physical Society under the terms of the Creative Commons Attribution 4.0 International license. Further distribution of this work must maintain attribution to the author(s) and the published article's title, journal citation, and DOI. Funded by SCOAP ${ }^{3}$. the corresponding minimizing functional, in analogy with the Gribov-Zwanziger approach in Landau gauge [1,2]. We start by reviewing how the minimal LCG can be fixed on the lattice, in Sec. II. We then consider the quadratic form obtained from the second variation of the LCG minimizing functional and its relation to the FP operator in the continuum formulation. First results for the ghost propagator in the LCG are shown in Sec. III for the SU(2) and $\mathrm{SU}(3)$ gauge groups. Finally, in the last section, we present our conclusions.

\section{MINIMAL LINEAR COVARIANT GAUGE}

The minimal LCG can be obtained [5] by minimizing the functional

$$
\begin{aligned}
\mathcal{E}_{\mathrm{LCG}}[U ; \Lambda ; h] \equiv & \Re \operatorname{Tr} \sum_{\vec{x} \in \Lambda_{x}}\{[i h(\vec{x}) \Lambda(\vec{x})] \\
& \left.-\sum_{\mu=1}^{d}\left[h(\vec{x}) U_{\mu}(\vec{x}) h\left(\vec{x}+\vec{e}_{\mu}\right)^{\dagger}\right]\right\},
\end{aligned}
$$

with the remark that, in the numerical minimization, the link variables $U_{\mu}(\vec{x})$ are gauge transformed, while the $\Lambda(\vec{x})$ matrices are not. The above definition applies to a $d$-dimensional Euclidean lattice $\Lambda_{x}$-usually with periodic boundary conditions - for an $\mathrm{SU}\left(N_{c}\right)$ gauge theory. Here, $\vec{e}_{\mu}$ is a vector of length $a$ in the positive $\mu$ direction, $a$ is the lattice spacing, the vectors $\vec{x}$ have components $x_{\mu} \in\{a, 2 a, \ldots, N a\}$ so that the lattice volume $V$ is equal to $N^{d}$, we indicate with $\operatorname{Tr}$ the trace in color space, $\Re$ selects the real part, and ${ }^{\dagger}$ stands for the Hermitian conjugate. Also, $\left\{U_{\mu}(\vec{x})\right\} \in \mathrm{SU}\left(N_{c}\right)$ is a given thermalized link configuration, and $\{h(\vec{x})\} \in \mathrm{SU}\left(N_{c}\right)$ is a gauge transformation. Both the $U_{\mu}(\vec{x})$ and $h(\vec{x})$ matrices are in the $N_{c} \times N_{c}$ (fundamental) representation. For the $N_{c}^{2}-1$ traceless Hermitian generators $\lambda^{b}$ of $\mathrm{SU}\left(N_{c}\right)$, we use the normalization $\operatorname{Tr}\left(\lambda^{b} \lambda^{c}\right)=2 \delta^{b c}$. 
Finally, $\Lambda(\vec{x}) \equiv \sum_{b} \Lambda^{b}(\vec{x}) \lambda^{b}$ are (Hermitian) matrices belonging to the $\mathrm{SU}\left(N_{c}\right)$ Lie algebra, and the $\Lambda^{b}(\vec{x})$ are random real numbers, usually Gaussian distributed around zero with a width $\sigma=\sqrt{\xi}$.

The first and second variations of $\mathcal{E}_{\mathrm{LCG}}[U ; h]$ can be obtained [11] by considering for the gauge transformation a one-parameter subgroup $h(\tau ; \vec{x}) \equiv \exp \left[i \tau \sum_{b} \gamma^{b}(\vec{x}) \lambda^{b}\right]$, where the parameter $\tau$ and the factors $\gamma^{b}(\vec{x})$ are real. Then, by expanding the functional $\mathcal{E}_{\mathrm{LCG}}[U ; \Lambda ; h](\tau)$ around a minimum $\left\{U_{\mu}(\vec{x})\right\}$ up to terms linear in $\tau$, and by using periodicity, one finds that the stationarity condition $\mathcal{E}_{\mathrm{LCG}}[U ; \Lambda ; h]^{\prime}(0)=0$-where ' indicates the derivative with respect to the parameter $\tau$-gives

$$
0=\Re \operatorname{Tr} \lambda^{b}\left[-\Lambda(\vec{x})+\sum_{\mu=1}^{d} \frac{U_{\mu}(\vec{x})-U_{\mu}\left(\vec{x}-\vec{e}_{\mu}\right)}{i}\right]
$$

for any lattice site $\vec{x}$ and color index $b$. One usually defines the lattice gauge field $A_{\mu}\left(\vec{x}+\vec{e}_{\mu} / 2\right)=\sum_{b} A_{\mu}^{b}\left(\vec{x}+\vec{e}_{\mu} / 2\right) \lambda^{b}$ through the relation

$$
\begin{aligned}
A_{\mu}\left(\vec{x}+\vec{e}_{\mu} / 2\right) \equiv & \frac{1}{2 i}\left[U_{\mu}(\vec{x})-U_{\mu}^{\dagger}(\vec{x})\right] \\
& -1 \frac{\operatorname{Tr}}{2 i N_{c}}\left[U_{\mu}(\vec{x})-U_{\mu}^{\dagger}(\vec{x})\right],
\end{aligned}
$$

where $1 \downarrow$ is the $N_{c} \times N_{c}$ identity matrix, yielding $A_{\mu}^{b}\left(\vec{x}+\vec{e}_{\mu} / 2\right)=\Re \operatorname{Tr}\left[\lambda^{b} U_{\mu}(\vec{x}) /(2 i)\right]$. Then, if we indicate with

$$
\begin{aligned}
\left(\nabla \cdot A^{b}\right)(\vec{x}) & \equiv \sum_{\mu=1}^{d}\left(\nabla_{\mu} A_{\mu}^{b}\right)(\vec{x}) \\
& \equiv \sum_{\mu=1}^{d} A_{\mu}^{b}\left(\vec{x}+\vec{e}_{\mu} / 2\right)-A_{\mu}^{b}\left(\vec{x}-\vec{e}_{\mu} / 2\right)
\end{aligned}
$$

the lattice divergence of the gauge field and we use $\nabla_{\mu}$ for the symmetrized lattice derivative, Eq. (2) becomes

$$
\left(\nabla \cdot A^{b}\right)(\vec{x})=\Lambda^{b}(\vec{x}) .
$$

We also define $U_{\mu}(\vec{x}) \equiv \exp \left[\operatorname{iag}_{0} \hat{A}_{\mu}\left(\vec{x}+\vec{e}_{\mu} / 2\right)\right]$, where $\hat{A}_{\mu}(\vec{x})$ is the continuum gauge field and $g_{0}$ is the bare coupling constant. Thus, in the limit of small $a$, we have that $A_{\mu}^{b}\left(\vec{x}+\vec{e}_{\mu} / 2\right)=a g_{0} \hat{A}_{\mu}^{b}\left(\vec{x}+\vec{e}_{\mu} / 2\right)+\mathcal{O}\left(a^{3} g_{0}^{3}\right)$, and a similar relation applies to $A_{\mu}^{b}(\vec{x})$. Note that, compared to the usual generators $\tilde{\lambda}^{b}$ with normalization $\operatorname{Tr}\left(\tilde{\lambda}^{b} \tilde{\lambda}^{c}\right)=\delta^{b c} / 2$, we have $\tilde{\lambda}^{b}=\lambda^{b} / 2$. This implies that $2 \hat{A}_{\mu}^{b}(\vec{x}) \approx$ $2 A_{\mu}^{b}(\vec{x}) /\left(a g_{0}\right)$ is the usual gauge field in the continuum limit. Also, in the formal continuum limit, i.e., $a \rightarrow 0$, $N \rightarrow+\infty$ with $L \equiv a N$ fixed, the above equation (5) becomes $a^{2} g_{0} \sum_{\mu=1}^{d}\left[\partial_{\mu} \hat{A}_{\mu}^{b}(\vec{x})+\mathcal{O}\left(a^{2}\right)\right]=\Lambda^{b}(\vec{x})$, which should be compared ${ }^{1}$ to the (usual) continuum gauge condition $2 \sum_{\mu} \partial_{\mu} \hat{A}_{\mu}^{b}(\vec{x})=\hat{\Lambda}^{b}(\vec{x})$; i.e., the continuum functions $\hat{\Lambda}^{b}(\vec{x})$ satisfy the relation $a^{2} g_{0} \hat{\Lambda}^{b}(\vec{x}) \approx 2 \Lambda^{b}(\vec{x})$. Moreover, since the lattice parameter $\beta$ is given by $2 N_{c} /\left(a^{4-d} g_{0}^{2}\right)$ in the $d$-dimensional case, by setting ${ }^{2}$

$$
\xi=\frac{N_{c} \hat{\xi}}{2 \beta},
$$

we have that

$$
\frac{1}{2 \xi} \sum_{x, b}\left[\Lambda^{b}(x)\right]^{2}=\frac{\beta /\left(2 N_{c}\right)}{2 \hat{\xi}} \sum_{x, b}\left[a^{2} g_{0} \hat{\Lambda}^{b}(x)\right]^{2}
$$

goes to $(2 \hat{\xi})^{-1} \int d^{d} x \sum_{b}\left[\hat{\Lambda}^{b}(x)\right]^{2}$ in the formal continuum limit. Thus, the continuum and lattice widths (of the corresponding Gaussian distributions) are related through the expression $\sigma=\hat{\sigma} \sqrt{N_{c} /(2 \beta)}$; i.e., for $N_{c}=2$, 3, one has $\sigma<\hat{\sigma}$ for typical values of $\beta$ in the scaling region.

In minimal Landau gauge, the FP matrix $\mathcal{M}^{b c}(\vec{x}, \vec{y})$ is obtained from the second-order expansion, with respect to the parameter $\tau$, of the corresponding minimizing functional, i.e., through the relations ${ }^{3}$

$$
\begin{gathered}
\frac{\mathcal{E}_{\mathrm{LG}}[U ; h]^{\prime \prime}(0)}{2}=\sum_{b, \vec{x}} \gamma^{b}(\vec{x})(\mathcal{M} \gamma)^{b}(\vec{x}) \\
(\mathcal{M} \gamma)^{b}(\vec{x})=\sum_{c, \vec{y}} \mathcal{M}^{b c}(\vec{x}, \vec{y}) \gamma^{c}(\vec{y}),
\end{gathered}
$$

where $\mathcal{E}_{\mathrm{LG}}[U ; h]$ is the Landau-gauge minimizing functional, given by the second term in the above Eq. (1).

On the other hand, one can easily verify that the first term in Eq. (1) does not contribute to this second-order expansion in powers of the parameter $\tau$. Indeed, the expression multiplying $\tau^{2}$ is given by

$$
\operatorname{Tr} \sum_{b, c, e, \vec{x}}\left[\gamma^{b}(\vec{x}) \gamma^{c}(\vec{x}) f^{b c e} \lambda^{e} \Lambda(\vec{x})\right]
$$

In the above derivation, we made use of the Hermiticity of the matrices $\lambda^{b}, \Lambda(\vec{x})$, and we employed the cyclic property of the trace and the commutation relations $\left[\lambda^{b}, \lambda^{c}\right] \equiv$ $2 i \sum_{e} f^{b c e} \lambda^{e}$, where $f^{b c e}$ are the (real) structure constants of the $\mathrm{SU}\left(N_{c}\right)$ gauge group. Let us recall that these structure constants are completely skew symmetric in all indices [12], since the Lie algebra of the $\mathrm{SU}\left(N_{c}\right)$ group is

\footnotetext{
${ }^{1}$ Here, notation and conclusions are different from Ref. [5].

${ }^{2}$ When one considers the usual generators $\tilde{\lambda}^{b}$, the relation between the lattice and continuum gauge parameters is $\xi=2 N_{c} \hat{\xi} / \beta$.

${ }^{3}$ From now on, we simplify the notation, and we do not indicate explicitly the lower and upper bounds for the summation indices.
} 
simple and compact, and the generators $\lambda^{b}$ constitute an orthonormal basis (through a global rescaling). Therefore, the expression $\sum_{b, c} \gamma^{b}(\vec{x}) \gamma^{c}(\vec{x}) f^{b c e}$ in Eq. (10) is zero $\forall e, \vec{x}$. As a consequence, the second variation of $\mathcal{E}_{\mathrm{LCG}}[U ; \Lambda ; h]$ yields the same matrix obtained in the Landau case, i.e., [11]

$$
\begin{aligned}
\mathcal{M}^{b c}(\vec{x}, \vec{y}) \equiv & \sum_{\mu}\left\{\Gamma_{\mu}^{b c}(\vec{x})\left[\delta_{\vec{x}, \vec{y}}-\delta_{\vec{x}+\vec{e}_{\mu}, \vec{y}}\right]\right. \\
& +\Gamma_{\mu}^{b c}\left(\vec{x}-\vec{e}_{\mu}\right)\left[\delta_{\vec{x}, \vec{y}}-\delta_{\vec{x}-\vec{e}_{\mu}, \vec{y}}\right] \\
& -\sum_{e} f^{b e c}\left[A_{\mu}^{e}\left(\vec{x}-\vec{e}_{\mu} / 2\right) \delta_{\vec{x}-\vec{e}_{\mu}, \vec{y}}\right. \\
& \left.\left.-A_{\mu}^{e}\left(\vec{x}+\vec{e}_{\mu} / 2\right) \delta_{\vec{x}+\vec{e}_{\mu}, \vec{y}}\right]\right\}
\end{aligned}
$$

with

$$
\Gamma_{\mu}^{b c}(\vec{x}) \equiv \operatorname{Tr}\left[\frac{\lambda^{b} \lambda^{c}+\lambda^{c} \lambda^{b}}{4} \frac{U_{\mu}(\vec{x})+U_{\mu}^{\dagger}(\vec{x})}{2}\right]
$$

It is immediate to verify that $\mathcal{M}^{b c}(\vec{x}, \vec{y})$ is symmetric under the simultaneous exchanges $b \leftrightarrow c$ and $\vec{x} \leftrightarrow \vec{y}$.

One can also set

$$
\begin{gathered}
\mathcal{M}=\frac{1}{2}\left(\mathcal{M}_{+}+\mathcal{M}_{-}\right) \\
\left(\mathcal{M}_{ \pm} \gamma\right)^{b}(\vec{x}) \equiv(\mathcal{M} \gamma)^{b}(\vec{x}) \pm(\Delta \mathcal{M} \gamma)^{b}(\vec{x}) \\
(\Delta \mathcal{M})^{b c}(\vec{x}, \vec{y}) \equiv \sum_{e} f^{b e c}\left(\nabla \cdot A^{e}\right)(\vec{x}) \delta_{\vec{x}, \vec{y}}
\end{gathered}
$$

At the same time, we define the lattice gauge-covariant derivative by the relation [11]

$$
\begin{aligned}
D_{\mu}^{b c}(\vec{x}, \vec{y}) \equiv & \Gamma_{\mu}^{b c}(\vec{x})\left[\delta_{\vec{x}+\vec{e}_{\mu}, \vec{y}}-\delta_{\vec{x}, \vec{y}}\right] \\
& -\sum_{e} f^{b e c} A_{\mu}^{e}\left(\vec{x}+\vec{e}_{\mu} / 2\right)\left[\delta_{\vec{x}+\vec{e}_{\mu}, \vec{y}}+\delta_{\vec{x}, \vec{y}}\right] .
\end{aligned}
$$

Indeed, in the formal continuum limit, we have $\Gamma_{\mu}^{b c}(\vec{x}) \rightarrow$ $\delta^{b c}+\mathcal{O}\left(a^{2} g_{0}^{2}\right)$, giving

$$
\left(D_{\mu} \gamma\right)^{b}(\vec{x}) \rightarrow a\left[\left(D_{\mu}[\hat{A}] \gamma\right)^{b}(\vec{x})+\mathcal{O}\left(a, a g_{0}\right)\right],
$$

where $D_{\mu}^{b c}[\hat{A}] \equiv \delta^{b c} \partial_{\mu}+2 g_{0} \sum_{e} f^{b c e} \hat{A}_{\mu}^{e}(\vec{x})$ is the continuum gauge-covariant derivative. [As explained above, with our notation, the continuum gauge field is given by $2 \hat{A}_{\mu}^{e}(\vec{x})$.] Then, it is easy to verify that

$$
\begin{aligned}
\left(\mathcal{M}_{+} \gamma\right)^{b}(\vec{x}) & =-\sum_{\mu}\left[\left(D_{\mu} \gamma\right)^{b}(\vec{x})-\left(D_{\mu} \gamma\right)^{b}\left(\vec{x}-\vec{e}_{\mu}\right)\right] \\
& \equiv-\sum_{\mu}\left[\nabla_{\mu}^{(-)}\left(D_{\mu} \gamma\right)\right]^{b}(\vec{x})
\end{aligned}
$$

where $\nabla_{\mu}^{(-)}$is the usual backward lattice derivative. Thus, $\mathcal{M}_{+}$is a lattice discretization of the continuum operator $\hat{\mathcal{M}}_{+}^{b c}[\hat{A}] \equiv-\sum_{\mu} \partial_{\mu} D_{\mu}^{b c}[\hat{A}]$, and we have $\mathcal{M}_{+}^{b c}=$ $a^{2}\left[\hat{\mathcal{M}}_{+}^{b c}[\hat{A}]+\mathcal{O}\left(a, a g_{0}\right)\right]$ in the limit $a \rightarrow 0$. Also, from the above Eq. (16), we can define the transpose lattice gaugecovariant derivative

$$
\begin{aligned}
\left(D_{\mu}^{T}\right)^{b c}(\vec{x}, \vec{y}) \equiv & \Gamma_{\mu}^{b c}\left(\vec{x}-\vec{e}_{\mu}\right) \delta_{\vec{x}-\vec{e}_{\mu}, \vec{y}}-\Gamma_{\mu}^{b c}(\vec{x}) \delta_{\vec{x}, \vec{y}} \\
& +\sum_{c, e} f^{b e c}\left[A_{\mu}^{e}\left(\vec{x}+\vec{e}_{\mu} / 2\right) \delta_{\vec{x}, \vec{y}}\right. \\
& \left.+A_{\mu}^{e}\left(\vec{x}-\vec{e}_{\mu} / 2\right) \delta_{\vec{x}-\vec{e}_{\mu}, \vec{y}}\right]
\end{aligned}
$$

which goes to $-a\left[D_{\mu}^{b c}[\hat{A}]+\mathcal{O}\left(a, a g_{0}, a g_{0}^{2}\right)\right]$ in the formal continuum limit. Then, one can verify that

$$
\left(\mathcal{M}_{-} \gamma\right)^{b}(\vec{x})=\sum_{\mu}\left[D_{\mu}^{T}\left(\nabla_{\mu}^{(+)} \gamma\right)\right]^{b}(\vec{x})
$$

where $\nabla_{\mu}^{(+)}$is the usual forward lattice derivative, and we can identify $\mathcal{M}_{-}$with a lattice discretization of the continuum operator $\hat{\mathcal{M}}_{-}^{b c}[\hat{A}] \equiv-\sum_{\mu} D_{\mu}^{b c}[\hat{A}] \partial_{\mu}$. Indeed, in the limit $a \rightarrow 0$, we have that $\mathcal{M}_{-}$goes to $a^{2}\left[\hat{\mathcal{M}}_{-}^{b c}[\hat{A}]+\mathcal{O}\left(a, a g_{0}, a g_{0}^{2}\right)\right]$. Finally, since the transpose of the backward lattice derivative $\nabla_{\mu}^{(-)}$is given by $-\nabla_{\mu}^{(+)}$, it is evident that $\mathcal{M}_{-}^{T}=\mathcal{M}_{+}$[and thus $\mathcal{M}_{+}^{T}=\mathcal{M}_{-}$]. Therefore, the matrix $\mathcal{M}$ in Eq. (13) can be written as $\left(\mathcal{M}_{+}+\mathcal{M}_{+}^{T}\right) / 2=\left(\mathcal{M}_{-}^{T}+\mathcal{M}_{-}\right) / 2$, which is clearly symmetric (and real), in agreement with the expression (11).

One should recall that, in the Landau case, the expression (15) is trivially null, due to the transversality condition $\left(\nabla \cdot A^{e}\right)(\vec{x})=0$, and one has that (on the lattice as well as in the continuum) $\mathcal{M}=\mathcal{M}_{+}=\mathcal{M}_{-}$. This is not the case in the LCG; the matrices $\mathcal{M}, \mathcal{M}_{+}$, and $\mathcal{M}_{-}$are different. However, since the expression $(15)$ for $(\Delta \mathcal{M})^{b c}(\vec{x}, \vec{y})$ is skew symmetric under the simultaneous exchanges $b \leftrightarrow c$ and $\vec{x} \leftrightarrow \vec{y}$, these matrices cannot be distinguished as quadratic forms. This is a general result; given a square matrix $M$, the corresponding quadratic form depends [13] only on its symmetric part $\left(M+M^{T}\right) / 2$. Thus, the FP matrix obtained from the second variation of a minimizing functional is defined modulo an arbitrary, additive skewsymmetric term. The situation is similar to the problem of defining a conserved energy-momentum tensor $T^{\mu \nu}$ in field theory [14], since the condition $\partial_{\mu} T^{\mu \nu}=0$ implies that $T^{\mu \nu}$ is defined modulo an additive term $\partial_{\rho} f^{\mu \nu \rho}$, with $f^{\mu \nu \rho}=-f^{\rho \nu \mu}$. This freedom is related to the freedom of adding to the Lagrangian a null (surface) divergence term, and it is usually employed to make the energy-momentum 
tensor symmetric and gauge invariant (in the case of a gauge theory). In our case, we can use the freedom of adding to the symmetric lattice FP matrix $\mathcal{M}$ the skewsymmetric term $\Delta \mathcal{M}$ in order to obtain the lattice FP matrix $\mathcal{M}_{+}=-\nabla^{(-)} \cdot D$, thus getting (in the limit $a \rightarrow 0$ ) the usual continuum result $-\sum_{\mu} \partial_{\mu} D_{\mu}^{b c}[\hat{A}]$. Equivalently, we could add to the minimizing functional $\mathcal{E}_{\mathrm{LCG}}[U ; \Lambda ; h]$ the null term $-\Re \operatorname{Tr} \sum_{\vec{x}} i[h(\vec{x}), \Lambda(\vec{x})] h(\vec{x})^{\dagger}$, which obviously does not affect the minimizing procedure. Indeed, by considering the one-parameter subgroup $h(\tau ; \vec{x})$ and by expanding the above expression at order $\tau^{2}$, we find-by a convenient reordering of the null terms and by using the stationarity condition (5) - the quadratic expression $\sum_{b, c, \vec{x}, \vec{y}} \gamma^{b}(\vec{x})(\Delta \mathcal{M})^{b c}(\vec{x}, \vec{y}) \gamma^{c}(\vec{y})$.

Let us note that, following the usual continuum FP approach, the matrix $\mathcal{M}_{+}$can also be obtained from a variation of the gauge condition (2) with respect to the gauge transformation $h(\vec{x}) \equiv \exp \left[i \sum_{b} \gamma^{b}(\vec{x}) \lambda^{b}\right]$, namely, by evaluating the functional derivative of

$$
\begin{aligned}
& \Re \operatorname{Tr} \lambda^{b}\left[-\Lambda(\vec{x})+i \sum_{\mu=1}^{d} h\left(\vec{x}-\vec{e}_{\mu}\right) U_{\mu}\left(\vec{x}-\vec{e}_{\mu}\right) h(\vec{x})^{\dagger}\right. \\
& \left.\quad-h(\vec{x}) U_{\mu}(\vec{x}) h\left(\vec{x}+\vec{e}_{\mu}\right)^{\dagger}\right]
\end{aligned}
$$

with respect to $\gamma^{c}(\vec{y})$. This adds a heuristic motivation for the consideration of $\mathcal{M}_{+}$among all the possible discretizations of the continuum FP matrix in LCG.

\section{GHOST PROPAGATOR}

In order to evaluate the ghost propagator

$$
G(k) \equiv \frac{1}{V} \sum_{b, \vec{x}, \vec{y}} e^{i \vec{k} \cdot \vec{x}}\left[\left(\mathcal{M}_{+}\right)^{-1}\right]^{b b}(\vec{x}, \vec{y}) e^{-i \vec{k} \cdot \vec{y}}
$$

in the LCG, we need to invert the FP matrix $\mathcal{M}_{+}$, defined in Eqs. (11), (14), and (15) above. Since this matrix is real but not symmetric, its eigenvalues and eigenvectors do not need to be real, and the nonreal eigenvalues and eigenvectors occur in complex-conjugate pairs. Also, one can easily check that only the symmetric (respectively, skew-symmetric) part of the inverse matrix $\left(\mathcal{M}_{+}\right)^{-1}$ contributes to the real (respectively, imaginary) part of the rhs of Eq. (22). Thus, the ghost propagator in the LCG is in general a complex quantity, while in Landau gauge it is always real. Finally, in order to invert $\mathcal{M}_{+}$, one cannot use, as in the Landau cause, the conjugate gradient method (since $\mathcal{M}_{+}$is not symmetric); i.e., one needs a more general iterative Krylov subspace method, applicable to generic nonsingular matrices [15].

We have performed tests evaluating the ghost propagator in the four-dimensional case, for the $\mathrm{SU}(2)$ and $\mathrm{SU}(3)$ gauge groups, using, respectively, the biconjugate gradient stabilized algorithm and the generalized conjugate residual for the inversion of the FP matrix [15]. For these two gauge
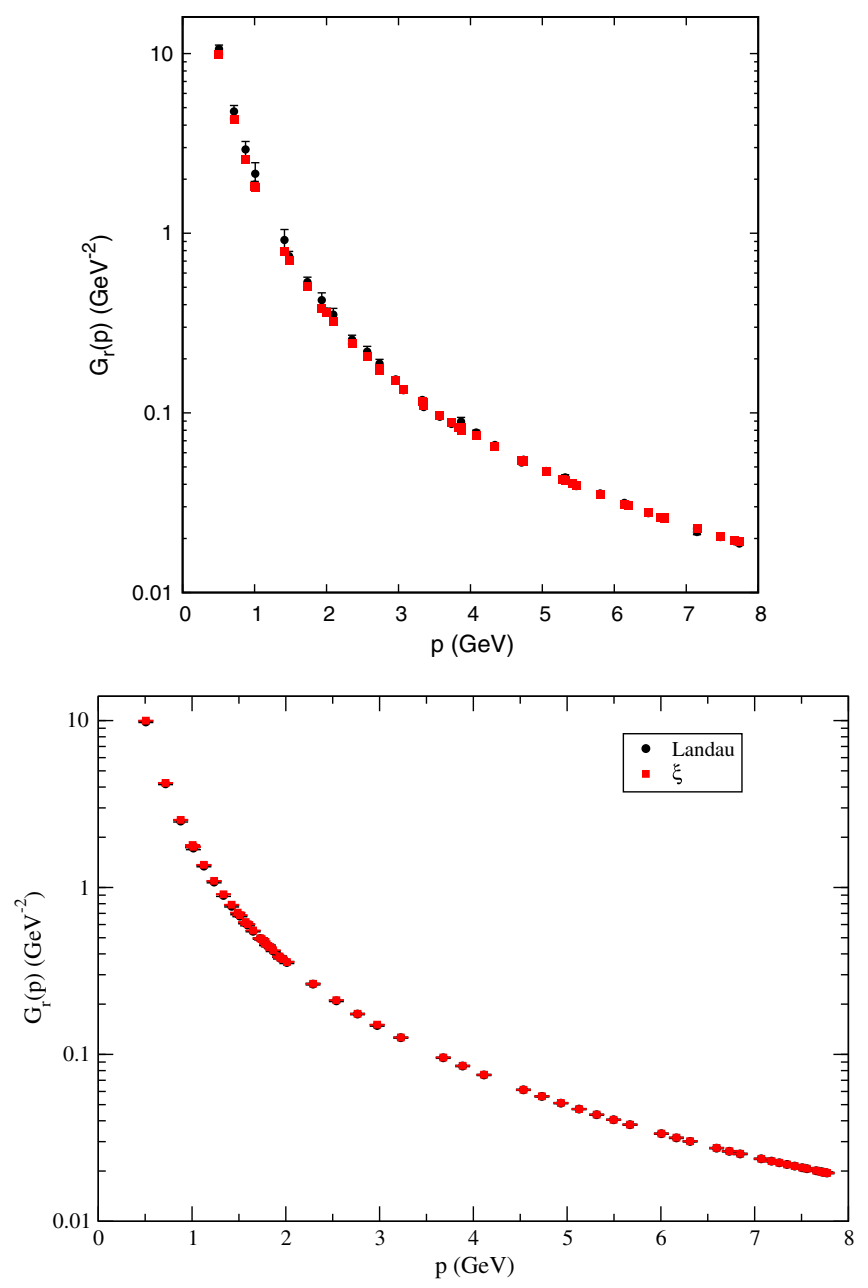

FIG. 1. The (real part of the) ghost propagator $G_{\mathrm{r}}(p)$ in the minimal LCG (filled rectangle) and in Landau gauge (filled circle), as a function of the lattice momentum $p$, with $p_{\mu}(k)=$ $2 \sin \left(\pi k_{\mu} / N\right)$ and $k_{\mu}=1,2, \ldots, N / 2$. Note the logarithmic scale on the $y$ axis. Both $G_{\mathrm{r}}(p)$ and $p$ are in physical units. Top: $\mathrm{SU}(2)$ case with $V=24^{4}, \beta=2.4469$ and $\xi=0.1$, corresponding to the continuum value $\hat{\xi}=0.24469$, for 60 thermalized configurations. Bottom: $\mathrm{SU}(3)$ case with $V=24^{4}, \beta=6.0$ and $\xi=\hat{\xi}=0.1$ for 79 thermalized configurations.

groups, we have considered lattice couplings $\beta=2.4469$ and $\beta=6.0$, respectively, which both correspond [16] to lattice spacing $a \approx 0.102 \mathrm{fm}$. Simulations have been done for lattice volumes $V=16^{4}$ and $24^{4}$. Thus, for the larger volume, the (nonzero) lattice momenta range from about $500 \mathrm{MeV}$ to about $7.7 \mathrm{GeV}$. For each thermalized gauge configuration, we have generated 20 sets of Gaussiandistributed $\{\Lambda(x)\}$ matrices, with variance ${ }^{4} \xi=0.1$ in the

\footnotetext{
${ }^{4}$ In the SU(2) case, we used the $\lambda^{b}$ generators with normalization $\operatorname{Tr}\left(\lambda^{b} \lambda^{c}\right)=2 \delta^{b c}$. For SU(3), we employed the $\tilde{\lambda}^{b}=\lambda^{b} / 2$ generators with normalization $\operatorname{Tr}\left(\tilde{\lambda}^{b} \tilde{\lambda}^{c}\right)=\delta^{b c} / 2$. Thus, in the former case, we have $\hat{\xi}=\beta \xi$ [see Eq. (6)], while in the latter, we find $\hat{\xi}=\beta \xi / 6$ [see the equation in Footnote 2].
} 
SU(2) case (corresponding to $\hat{\xi}=0.24469$ ) and $\xi=\hat{\xi}=$ $0.1,0.2$ and 0.3 in the SU(3) case. ${ }^{5}$ The ghost propagator has been evaluated using a point source for the inversion [17]. Results are reported in Fig. 1, where we compare the real part of the ghost propagator $G_{\mathrm{r}}(p)$ in the minimal LCG with the corresponding data in Landau gauge, using the same set of thermalized configurations. Clearly, the data in the LCG are in agreement, within error bars, with the data in Landau gauge. Let us mention that, in continuum analytic works, one usually finds that $G(p)$ is real $[6,7,10,18]$. A numerical check of this result is postponed to a future study.

\section{CONCLUSIONS}

In this work, we have discussed the relation among the FP matrix in lattice minimal LCG and the second variation of the corresponding minimizing functional, following the usual Gribov-Zwanziger approach for Landau gauge [1,2]. In particular, we have chosen the matrix $\mathcal{M}_{+}$[see Eqs. (11), (14), and (15) above] as a natural lattice discretization of the LCG continuum FP operator $-\sum_{\mu} \partial_{\mu} D_{\mu}^{b c}[\hat{A}]$. We have also carried out some tests for the numerical inversion of the matrix $\mathcal{M}_{+}$and evaluated the ghost propagator. Preliminary results for the (real part of the) ghost propagator $G_{\mathrm{r}}(p)$ show no detectable difference with the corresponding lattice data in Landau gauge. Of course, numerical simulations for larger physical volumes,

\footnotetext{
${ }^{5}$ For $V=24^{4}$, in the $\mathrm{SU}(3)$ case, simulations have been done only for $\xi=\hat{\xi}=0.1$. These are the data shown in the bottom plot of Fig. 1. (Results for the $V=16^{4}$ cases are similar.)
}

different lattice spacings $a$, and different gauge parameters $\xi$ should be performed before any final conclusion is drawn about the behavior in the minimal LCG of $G_{\mathrm{r}}(p)$ at small momenta. One should also recall that, in the continuum, there are different possible setups for the ghost sector in the LCG (see e.g., Appendix A in Ref. [1]). The FP matrix $\mathcal{M}_{+}$, considered here, corresponds to the usual choice of complex ghost/antighost fields, without enforcing the ghost-antighost symmetry, which is naturally realized in Landau gauge. On the other hand, for a generic linear covariant gauge with $\xi \neq 0$, this choice is at odds with demanding Hermiticity of the underlying Lagrangian, which requires in principle the introduction of a doublet of real ghost/antighost fields [1,19]. Clearly, it would be important to analyze if and how the other setups can also be implemented on the lattice in minimal LCG. Another open question is how to define an appropriate Gribov region, similarly to the Landau-gauge case. A more detailed analysis of these issues will be presented elsewhere.

\section{ACKNOWLEDGMENTS}

A. C. and T. M. acknowledge partial support from CNPq. A.C. also acknowledges partial support from FAPESP (Grant No. 16/22732-1). The research of D. D. and M. R. is supported by KU Leuven IF Project No. C14/16/067. O. O. and P. J.S. acknowledge the Laboratory for Advanced Computing at University of Coimbra (http://www.uc. pt/ lca) for providing access to the HPC computing resource NAVIGATOR. P. J. S. acknowledges support by FCT under Contracts No. SFRH/BPD/40998/2007 and No. SFRH/ BPD/109971/2015. The SU(3) simulations were done using Chroma [20] and PFFT [21] libraries.
[1] R. Alkofer and L. von Smekal, Phys. Rep. 353, 281 (2001).

[2] J. Greensite, Lect. Notes Phys. 821, 1 (2011); N. Vandersickel and D. Zwanziger, Phys. Rep. 520, 175 (2012); A. Maas, Phys. Rep. 524, 203 (2013).

[3] D. Binosi and J. Papavassiliou, Phys. Rep. 479, 1 (2009); P. Boucaud, J. P. Leroy, A. Le Yaouanc, J. Micheli, O. Pène, and J. Rodríguez-Quintero, Few-Body Syst. 53, 387 (2012).

[4] L. Giusti, M. L. Paciello, S. Petrarca, C. Rebbi, and B. Taglienti, Nucl. Phys. B, Proc. Suppl. 94, 805 (2001); A. Cucchieri, A. Maas, and T. Mendes, Comput. Phys. Commun. 180, 215 (2009); P. Bicudo, D. Binosi, N. Cardoso, O. Oliveira, and P. J. Silva, Phys. Rev. D 92, 114514 (2015).

[5] A. Cucchieri, T. Mendes, and E. M. S. Santos, Phys. Rev. Lett. 103, 141602 (2009).

[6] M. Q. Huber, Phys. Rev. D 91, 085018 (2015).

[7] A. C. Aguilar, D. Binosi, and J. Papavassiliou, Phys. Rev. D 91, 085014 (2015).
[8] M. A. L. Capri, A. D. Pereira, R. F. Sobreiro, and S. P. Sorella, Eur. Phys. J. C 75, 479 (2015); F. Siringo and G. Comitini, Phys. Rev. D 98, 034023 (2018).

[9] F. Siringo, Phys. Rev. D 90, 094021 (2014).

[10] M. A. L. Capri, D. Fiorentini, M. S. Guimaraes, B. W. Mintz, L. F. Palhares, S. P. Sorella, D. Dudal, I. F. Justo, A. D. Pereira, and R. F. Sobreiro, Phys. Rev. D 93, 065019 (2016).

[11] D. Zwanziger, Nucl. Phys. B412, 657 (1994).

[12] J. F. Cornwell, Group Theory in Physics (Academic Press Limited, London, 1984).

[13] K. M. Abadir and J. R. Magnus, Matrix Algebra (Cambridge University Press, Cambridge, New York, 2005).

[14] P. Ramond, Field Theory: A Modern Primer (Benjamin/ Cummings, Reading, 1981), second printing with revisions; C. G. Callan, Jr., S. R. Coleman, and R. Jackiw, Ann. Phys. (N.Y.) 59, 42 (1970). 
[15] Y. Saad, Iterative Methods for Sparse Linear Systems, 2nd ed. (Society for Industrial and Applied Mathematics, Philadelphia, 2003).

[16] See A. Cucchieri, T. Mendes, O. Oliveira, and P. J. Silva, Phys. Rev. D 76, 114507 (2007) and references therein.

[17] P. Boucaud, J. P. Leroy, A. Le Yaouanc, A. Y. Lokhov, J. Micheli, O. Pène, J. Rodríguez-Quintero, and C. Roiesnel, Phys. Rev. D 72, 114503 (2005).
[18] A. I. Davydychev, P. Osland, and O. V. Tarasov, Phys. Rev. D 54, 4087 (1996); 59, 109901(E) (1999).

[19] T. Kugo and I. Ojima, Prog. Theor. Phys. Suppl. 66, 1 (1979).

[20] R. G. Edwards, and B. Joó (SciDAC, LHPC, and UKQCD Collaborations), Nucl. Phys. B, Proc. Suppl. 140, 832 (2005).

[21] M. Pippig, SIAM J. Sci. Comput. 35, C213 (2013). 